\title{
Influence of fines on the compression behaviour of decomposed volcanic rocks
}

\author{
Ismail Adeniyi Okewale $e^{1,2^{*}}$
}

\author{
${ }^{*}$ Correspondence: \\ iaokewale@futa.edu.ng \\ ${ }^{1}$ Federal University \\ of Technology Akure, P.M.B \\ 704, Akure, Nigeria \\ Full list of author information \\ is available at the end of the \\ article
}

\begin{abstract}
The compression behaviour of mixtures of sand with fines derived from decomposed volcanic rocks has been investigated in details. Oedometer tests were carried out on the samples of different degrees of weathering. The data show that no unique normal compression lines can be identified. The soils have medium degree of convergence and the degree of convergence is reducing with increasing fines. An attempt was made to investigate the particle breakage to further highlight the compression behaviour in a quantitative way. Soils with similar initial gradings have different particle breakage as a result of different degrees of weathering and the breakage can be linked to the quantity of larger particles. The particle breakage reduces as the amount of fines increases for the soils and when analysed the particle breakage further, it was observed that the more weathered volcanic samples have weaker particles.
\end{abstract}

Keywords: Fines content, Weathering, Compression, Transitional behaviour, Particle breakage

\section{Introduction}

Numerous studies have been carried out on the influence of fines, mineralogy and particle breakage on the mixtures of soils consisting of different grain size and types (e.g. [18, $22,26,28])$. Also, extensive laboratory investigations have been made into the mechanics of influence of fines on the behaviour of sand compared to weathered geomaterials (e.g. $[2,11,23,24])$. This may be due to the presence of sand in many areas around the world. However, weathered soils are abundant in tropical and subtropical areas of the world (e.g. West Africa, South America and South East Asia) and in Hong Kong particularly, decomposed igneous (granitic and volcanic) soils occurred in large quantity and engineering structures are built in and on them. Also, these weathered geomaterials are always encountered by practising engineers in their daily activities.

Despite the fact that several works have been carried out on the mechanics of behaviour of adding fines to sand, there are still some disagreements on the behaviour of binary mixtures of sand. For example, while it is less clear what the values of transitional fines should be for different soils and how the nature of the fines influences the mechanics of compression behaviour of soils studied by Zuo and Baudet [28], Lade and Yamamuro [11] and Carrera et al. [2] found that as the fines increase, the minimum value of compressibility and the lowest location of normal compression line in the $\mathrm{v}$ : lnp' plane being a transitional mixture. Also, Xiao et al. [24] investigated the mechanics 
of behaviour of well-graded coarse granular soil in drained shearing and found that the behaviour depended on the initial specific volumes.

Compared to other geomaterials, it is more difficult to characterise weathered soils due to high variability in their particle mineralogy, size, shape and distributions $[15$, $16,21]$ and more studies are required to shed light on their behaviour. The investigation into the influence of fines on the mechanics of weathered soils in compression is very few and very scarce for soils resulting from volcanic rock. While Okewale and Coop [15] investigated the effects of weathering on the physical properties, mechanical behaviour and geological structure of decomposed volcanic rocks and Okewale and Coop [16] studied different approaches to predict and analyse the behaviour of decomposed volcanics, this work presents the influence of fines on the mechanics of behaviour of decomposed volcanic soils in compression. This work is however different from the study of Xiao et al. [24] which investigated transitional behaviour in a well graded coarse granular soil with very small fines content (about 1.8\%) and large amount of large size particles $(93 \%>2.36 \mathrm{~mm})$ in shearing. Specifically, they studied the critical state behaviour of coarse granular soil. In this study, the fines contents are very large (10-30\%) and the objective is to show the effects of fines on the compression behaviour of decomposed volcanics with large particles $(0.3-2.36 \mathrm{~mm})$ far smaller than those of natural soil of Xiao et al. [24]. Also, particle breakage is investigated to shed more light on the compression behaviour of volcanic soils of different weathering degrees. This was achieved by carrying out oedometer tests on different mixtures of samples of two degrees of weathering from different depths and locations of Hong Kong Island.

\section{Materials and methodology}

The materials tested in this work were decomposed volcanic rocks belonging to grade IV (Highly Decomposed, HDV) and grade V (Completely Decomposed, CDV) according to international standards (e.g., [9]). According to Geotechnical Engineering Office [7] classification system of Hong Kong which is also based on international standards, samples were classified as weak to medium weak Highly Decomposed Volcanic rocks and weak Completely Decomposed Volcanic rocks. Due to the heterogeneous nature of decomposed volcanics, the HDV were from shallow depth (0.7-1 $\mathrm{m}$ ) and the CDV were from relatively deeper depth (13.3-13.95 m). The samples belonged to Ap Lei Chau formation which composed of fine-grained ash tuffs and taken from Hong Kong Island as presented in Fig. 1. The details of the characteristics of the original samples are given in Table 1.

Soils from the two weathering degrees were sieved into different sizes. The grain sizes are: $<0.063 \mathrm{~mm}, 0.063-0.15 \mathrm{~mm}, 0.15-0.30 \mathrm{~mm}, 0.30-0.60 \mathrm{~mm}, 0.60-1.18 \mathrm{~mm}, 1.18-$ $2.36 \mathrm{~mm}$ and $2.36-5 \mathrm{~mm}$. The grain size of less than $0.063 \mathrm{~mm}$ is referred to as fines in this paper, which are basically the mixture of clay and silt fractions but were plastic overall. The coarse fractions are referred to as sand for simplicity. The coarse grains used in this study ranged between 0.3 and $5 \mathrm{~mm}$. The sand proportions were prepared by mixing different gradings in equal proportions. Samples were prepared by mixing the fines and sand in different percentages. The mixtures tested were: $10 \%$ fines-90\% sand indicated as 1090, 20\% fines-80\% sand indicated as 2080 and 30\% fines-70\% sand indicated 


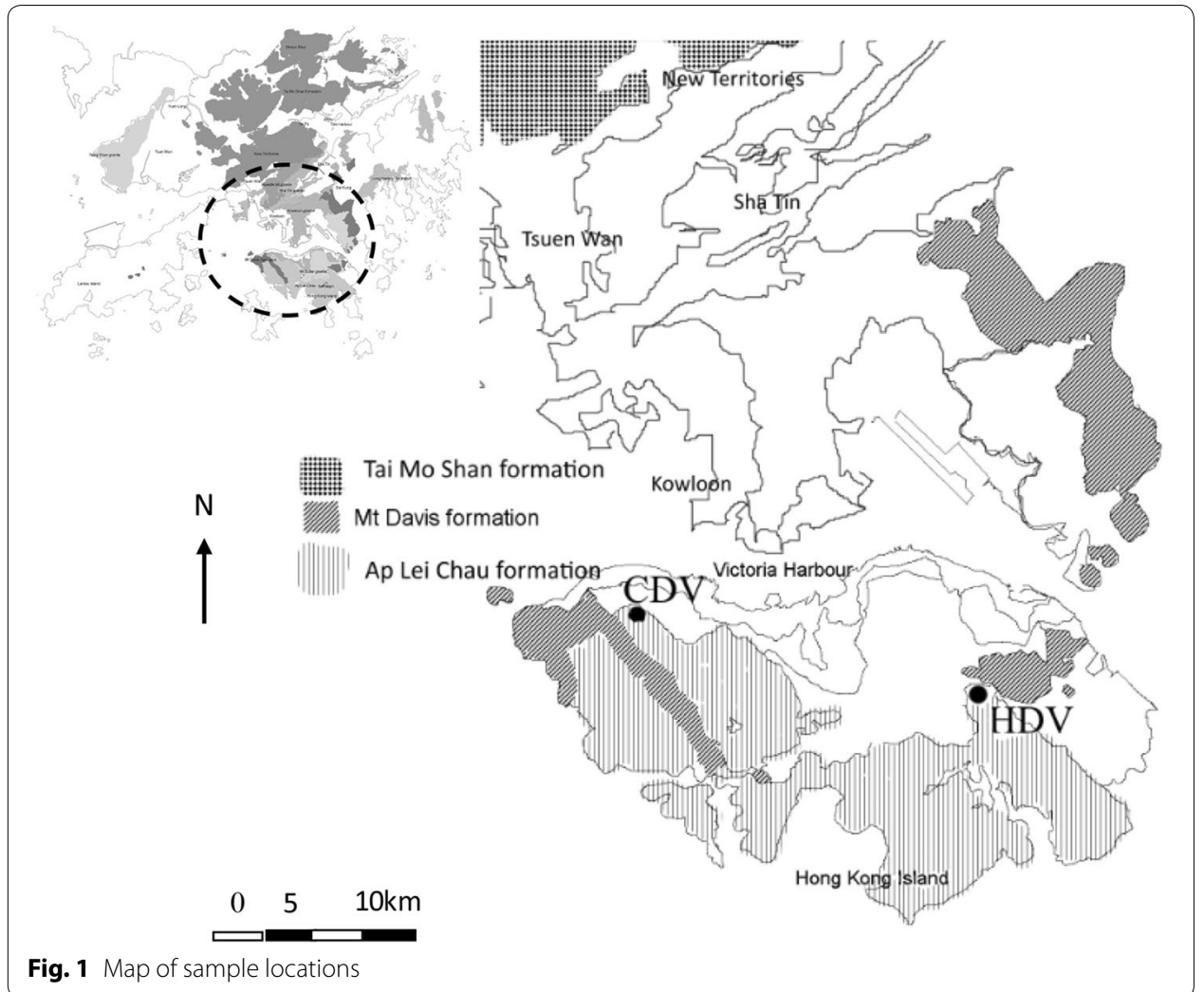

Table 1 Details of the original samples

\begin{tabular}{|c|c|c|c|c|c|c|c|c|c|c|c|c|}
\hline \multirow{2}{*}{$\begin{array}{l}\text { Weathering } \\
\text { degree }\end{array}$} & \multirow[t]{2}{*}{ Depth (m) } & \multirow[t]{2}{*}{$\mathbf{v}$} & \multirow[t]{2}{*}{ LL (\%) } & \multirow[t]{2}{*}{ PL (\%) } & \multirow[t]{2}{*}{ PI (\%) } & \multirow[t]{2}{*}{$\mathrm{D}_{50}(\mathrm{~mm})$} & \multirow[t]{2}{*}{$F_{c}(\%)$} & \multirow[t]{2}{*}{$C_{u}$} & \multirow[t]{2}{*}{$C_{c}$} & \multicolumn{3}{|c|}{ Mineralogy } \\
\hline & & & & & & & & & & $\mathrm{Cl}$ & $\mathbf{Q}$ & $\mathrm{Fe}$ \\
\hline DV & $13.3-13.95$ & 1.62 & 31.2 & 22 & 9.2 & 0.4 & 34 & 400 & 1.0 & 14 & 34 & 52 \\
\hline HDV & $0.7-1$ & 2.07 & 43.2 & 33.1 & 10.1 & 0.08 & 48 & 300 & 0.08 & 11 & 49 & 40 \\
\hline
\end{tabular}

$v$ specific volume, $L L$ liquid limit, $P L$ plastic limit, $P I$ plasticity index, $D_{50}$ mean particle size, $F_{c}$ fines content, $C_{u}$ coefficient of uniformity, $C_{c}$ coefficient of curvature, $C l$ clay, $Q$ quartz, Fe feldspar

Table 2 Details of the soils tested

\begin{tabular}{llllll}
\hline Soil & $\mathbf{D}_{\mathbf{5 0}}(\mathbf{m m})$ & $\mathbf{F}_{\mathbf{c}}(\%)$ & $\mathbf{C}_{\mathbf{u}}$ & $\mathbf{C}_{\mathbf{c}}$ & $\mathbf{I}_{\mathbf{G S}}$ \\
\hline 1090 & 0.50 & 10 & 11.11 & 1.41 & 0.045 \\
2080 & 0.41 & 20 & 13.49 & 0.26 & 0.030 \\
3070 & 0.32 & 30 & 16.03 & 0.06 & 0.019 \\
\hline 1090 10\% fines and 90\% sand, 2080 20\% fines and 80\% sand, 3070 30\% fines and 70\% sand, $\mathbf{I}_{\text {GS }}$ grain size index
\end{tabular}

as 3070 for the two weathering degrees. The details of the samples tested are given in Table 2.

Figure 2 presents the grading curves of original samples determined from wet sieving and sedimentation technique and artificial mixtures of CDV and HDV determined using dry and wet sieving due to the nature of the samples. The original samples are well-graded but due to heterogeneous nature of weathering processes and different 


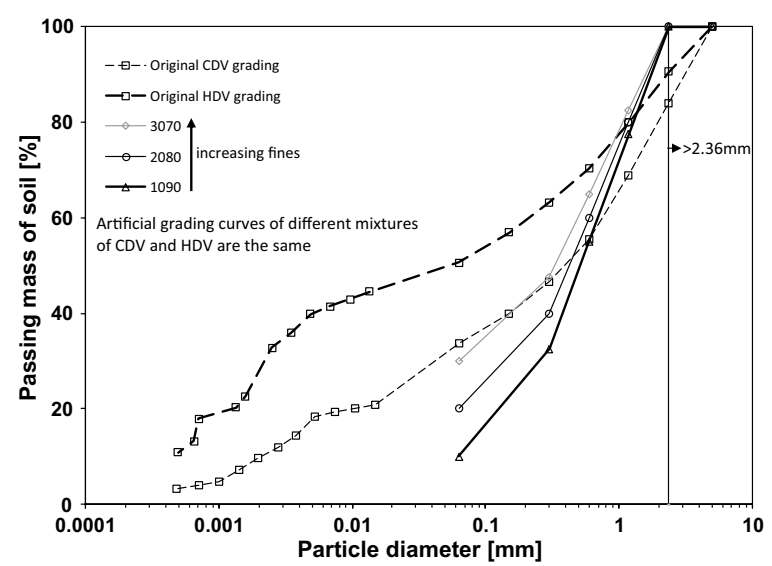

Fig. 2 Particle size distributions for the original soils and different mixtures of CDV and HDV

grain sizes of the parent rocks, the HDV plots above the CDV. The soils originating from igneous rocks do not undergo sorting in their grading, thereby resulting in wellgraded particle size distributions. The details of the in situ specific volumes, the grading descriptors determined from particle size distributions, the plasticity obtained from Atterberg limits [1] and mineralogy obtained from X-ray diffraction are given in Table 1. The particle size distribution curves for different mixtures of CDV and HDV are the same as presented in Fig. 2. As expected, the sample with more fines and low sand contents generally plots above the others. The engineering grading descriptors that define the shape and location individually and those that combined shape and location together are presented in Table 2 . The mean particle size is reducing with fines and the well-graded nature is increasing with increasing fines.

The samples were prepared by slowly adding de-aired water and mixed to form homogenous soil paste (slurry) with no particle segregation. The bowl containing the slurry was put in a vacuum chamber for more than $60 \mathrm{~min}$ in order to remove air bubbles and later vibrated to encourage an air bubbles to come out. The sample was then placed in the ring for the test. Different initial specific volumes were obtained by adding different amounts of de-aired water.

A Phillips XL30 FEG Environmental Scanning Electron Microscope (ESEM) equipped with energy dispersive spectrometer EDS was used to investigate the microstructure of the samples. The samples can be studied in either dry or wet conditions and this is one of the advantages of environmental SEM over the conventional SEM. The samples tested were taken from the broken surface so that a true reflection of the fabric can be seen. Figure 3 shows the ESEM micrographs of the samples of two degrees of weathering studied in this work. The micrographs are for a $50 \mu \mathrm{m}$ field of view for both weathering degrees. The fabrics are dominated by agglomeration of flat particles together to form continuous clusters. The CDV appears coarser grained (Fig. 3a) compared to the HDV (Fig. 3b) which confirms the gradings obtained from sieve analysis as earlier discussed. The fabric seems relatively homogenous but the clusters give some heterogeneity resulting in slight orientation of fabric. 

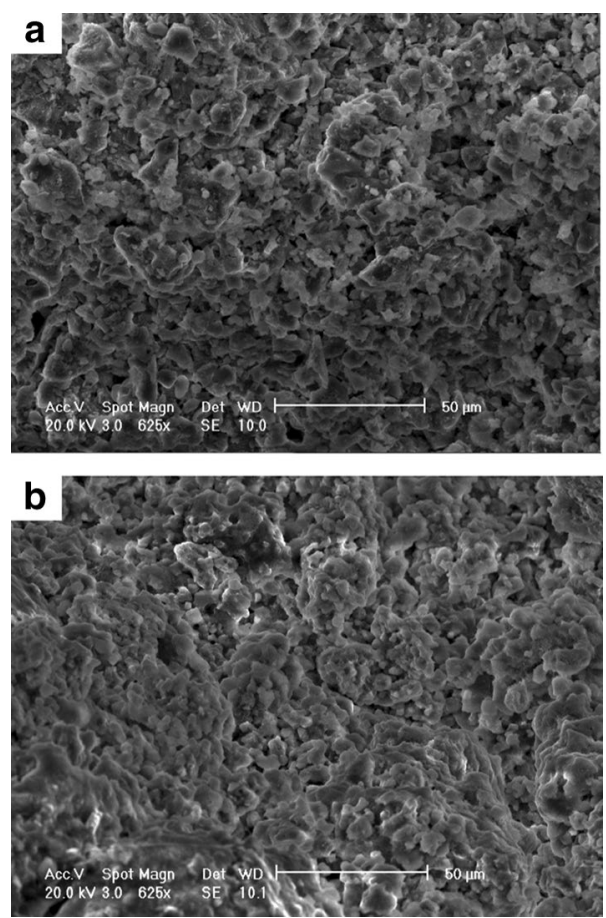

Fig. 3 SEM images; $\mathbf{a}$ CDV sample and $\mathbf{b}$ HDV sample

Table 3 Details of the oedometer tests

\begin{tabular}{|c|c|c|c|c|}
\hline Weathering degree & Sample tested & Test description & $v_{0}$ & Accuracy of $v( \pm)$ \\
\hline \multirow[t]{9}{*}{ CDV } & 3070 & C3070-1 & 1.74 & 0.02 \\
\hline & & C3070-2 & 2.25 & 0.02 \\
\hline & & C3070-3 & 1.65 & 0.005 \\
\hline & 2080 & C2080-1 & 1.61 & 0.03 \\
\hline & & C2080-2 & 1.83 & 0.03 \\
\hline & & C2080-3 & 1.77 & 0.02 \\
\hline & 1090 & C1090-1 & 1.90 & 0.007 \\
\hline & & C1090-2 & 2.02 & 0.05 \\
\hline & & C1090-3 & 1.77 & 0.006 \\
\hline \multirow[t]{9}{*}{ HDV } & 3070 & H3070-1 & 2.01 & 0.01 \\
\hline & & H3070-2 & 2.68 & 0.05 \\
\hline & & H3070-3 & 2.00 & 0.009 \\
\hline & 2080 & H2080-1 & 2.00 & 0.02 \\
\hline & & $\mathrm{H} 2080-2$ & 2.53 & 0.006 \\
\hline & & H2080-3 & 2.05 & 0.002 \\
\hline & 1090 & H1090-1 & 2.11 & 0.006 \\
\hline & & H1090-2 & 2.46 & 0.05 \\
\hline & & H1090-3 & 2.12 & 0.03 \\
\hline
\end{tabular}

$v_{o}$ initial specific volume, $v$ specific volume, $C$ completely decomposed, $H$ highly decomposed

Oedometer tests were conducted on the HDV and CDV mixtures using front loading oedometers. The details of the tests are given in Table 3. In the test descriptions, the first letter represents weathering degree, followed by the sample mixtures and 
finally the test number. A maximum load of $130 \mathrm{~kg}$ was applied which allowed a stress of up to $7 \mathrm{MPa}$ to be achieved using a $50 \mathrm{~mm}$ diameter and $20 \mathrm{~mm}$ height ring. The rings were designed and manufactured in order to optimise the accuracy of the measured specific volumes. The closed base rings were used because of its advantages over the convention double drainage rings. It does not require filter paper or porous stone at the base which can be a source of error in the measurement of specific volume. It also prevents loss of water from the base after draining the water bath at the end of the test. In addition, it allows the moist weight of the samples to be measured at the beginning and after the test without causing disturbance. However, excess pore water pressure could be generated as a result of poor drainage but it is assumed that pore pressures have been dissipated at every loading stage so that $\Delta \mathrm{u}=\mathrm{u}_{\mathrm{o}}=0$ and $\sigma_{\mathrm{v}}=\sigma_{\mathrm{v}}{ }^{\prime}$.

The specific volumes were calculated by careful measurements using different methods so as to improve the confidence, following the methods outlined in Rocchi and Coop [20] and Shipton and Coop [22]. The initial specific volume of each sample was derived from the sample weight, initial dimensions and water content while the final $\mathrm{v}$ was estimated from final measurements, back calculating the initial value using the volumetric strain measured in the test. Volumetric strain is the overall measured settlement divided by the average initial height. Three different equations were used and they were chosen to be as independent as possible:

$$
\begin{aligned}
& v_{i}=\frac{\gamma_{w}\left(1+w_{i}\right) G_{s}}{\gamma_{b i}} \\
& v_{i}=\frac{\gamma_{w}\left(1+w_{f}\right) G_{s}}{\gamma_{b f}\left(1-\varepsilon_{v o l}\right)} \\
& v_{i}=\frac{w_{f} G_{s}+1}{\left(1-\varepsilon_{v o l}\right)}
\end{aligned}
$$

where $v_{i}$ is the initial specific volume, $G_{s}$ specific gravity, $w_{i}$ initial water content, $w_{f}$ final water content, $\gamma_{w}$ the unit weight of water, $\gamma_{b i}$ initial bulk unit weight, $\gamma_{b f}$ final bulk unit weight and $\varepsilon_{v o l}$ is the volumetric strain.

Equations 1 and 2 are based on indirect measurement of the sample dry unit weight based on moist weight and water content irrespective of saturation degree and can both be used at the beginning and end of the test respectively. Equation 1 is based on initial measurements, that is, initial water content, $w_{i}$, and initial bulk unit weight, $\gamma_{b i}$, while Eq. 2 is based on final measurements, that is, final water content, $w_{f}$, final bulk unit weight, $\gamma_{b f}$, and volumetric strain, $\varepsilon_{v o l}$. In Eq. 2, the final measurements are used to back calculate initial $v$ using overall volumetric strain measured during the test. Also, Eq. 3 is based on $\mathrm{w}_{\mathrm{f} \text { and }} \varepsilon_{\mathrm{vol}}$ assuming the sample is fully saturated and the initial $\mathrm{v}$ is also obtained through back calculation. Since the samples used were prepared in slurry, the fully saturation assumption in the test is safe. A mean was taken for the various values of the initial specific volumes and the accuracy of the measurement was estimated by taking the largest difference between individual value and the mean. The estimated accuracies for the tests are also given in Table 3. 
Table 4 Typical example of calculation of initial specific volume

\begin{tabular}{lllllll}
\hline HDV & Test & \multicolumn{3}{l}{ Equation of $v_{i}$} & Mean $v_{i}$ & Estimated accuracy \\
\cline { 3 - 6 } & & $\frac{\gamma_{w}\left(1+w_{i}\right) G_{s}}{\gamma_{b i}}$ & $\frac{\gamma_{w}\left(1+w_{f}\right) G_{s}}{\gamma_{b f}\left(1-\varepsilon_{v o l}\right)}$ & $\frac{w_{f} G_{s}+1}{\left(1-\varepsilon_{v o l}\right)}$ & & \\
\hline 3070 & H3070-1 & 1.9990 & 2.0227 & 1.9964 & 2.01 & \pm 0.01
\end{tabular}

$v_{i}$ initial specific volume, $G_{s}$ specific gravity, $w_{i}$ initial water content, $w_{f}$ final water content, $\gamma_{w}$ the unit weight of water, $\gamma_{b i}$ initial bulk unit weight, $\gamma_{b f}$ final bulk unit weight and $\varepsilon_{v o l}$ is the volumetric strain

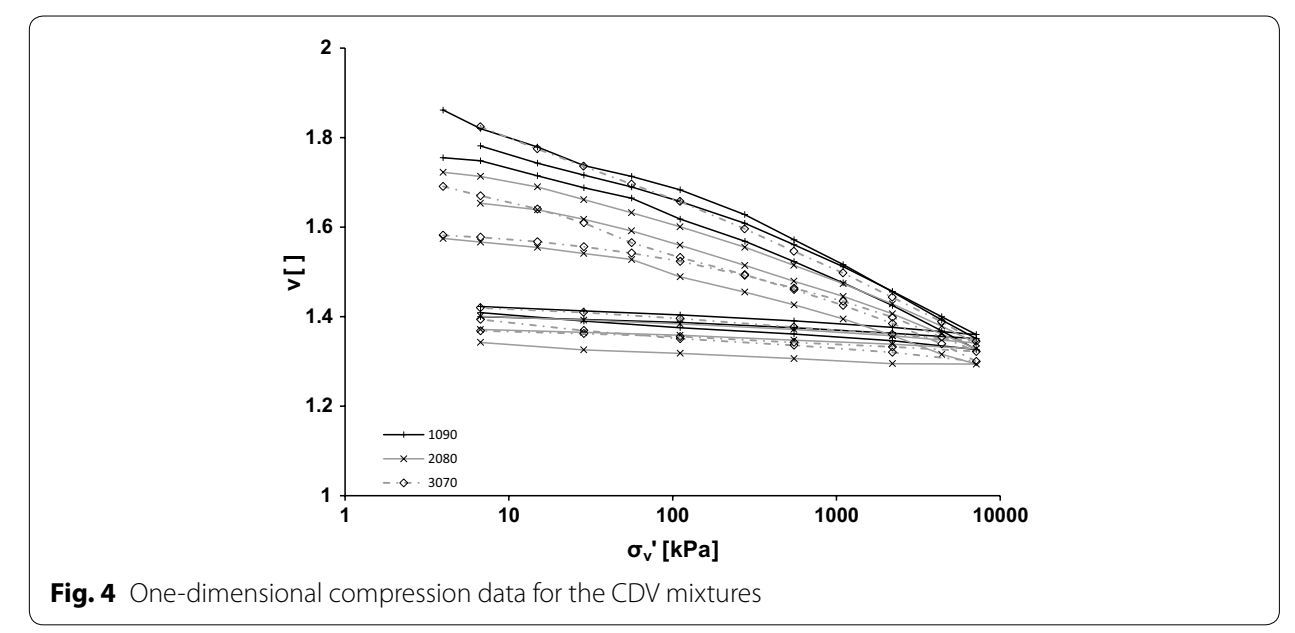

Table 4 shows a typical example of how the initial specific volume was calculated using three different equations for the HDV sample. The three equations were chosen to be as independent of each other as possible. The values are different for different equations.

\section{Results and discussions}

Figure 4 presents the oedometer test results from the CDV samples with different fines and sand mixtures. The samples were prepared to have different initial specific volumes. For each soil, a number of tests were conducted covering a wide range of initial specific volumes. The range of initial specific volumes that could be achieved varied for the soils owing to differences in their initial grading. The accuracy of initial specific volume is variable but generally good. At the highest stress reached in the tests, it is not possible to identify a unique one-dimensional normal compression lines (NCL) for the different mixtures, indicating that the compression paths do not converge to a unique NCL. The behaviour of different mixtures of the HDV in one-dimensional compression is shown in Fig. 5. A direct similar behaviour to the compression behaviour of different mixtures of CDV is observed. A similar behaviour of mixtures of different degrees of weathering may possibly results from the same mechanics of compression of constituent materials.

Although fines and sand compress as a result of different physical mechanisms, with physico-chemical forces play a significant role in the former and the stress being transmitted by direct grain contacts in the latter, the combined effects of both result in similar behaviour for different degrees of weathering. This is an indication of possible transitional mode of behaviour in the mixtures of samples of different degrees of weathering similar to what has been found for natural, reconstituted and artificial 


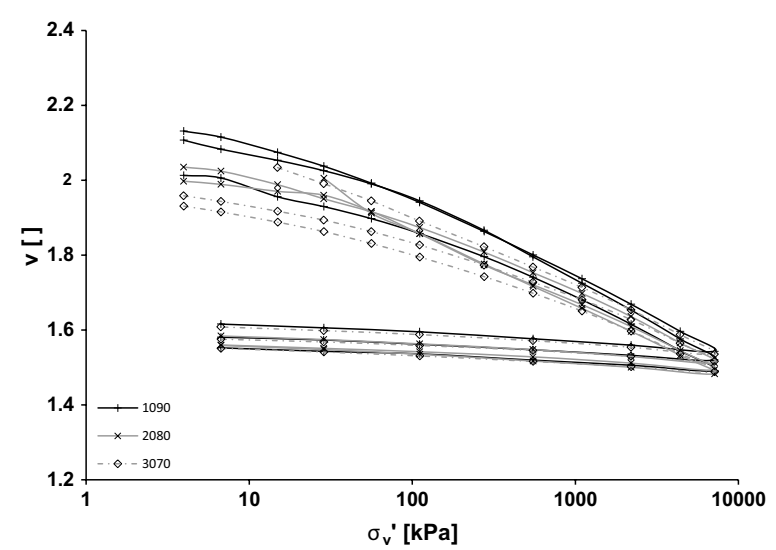

Fig. 5 One-dimensional compression data for the HDV mixtures
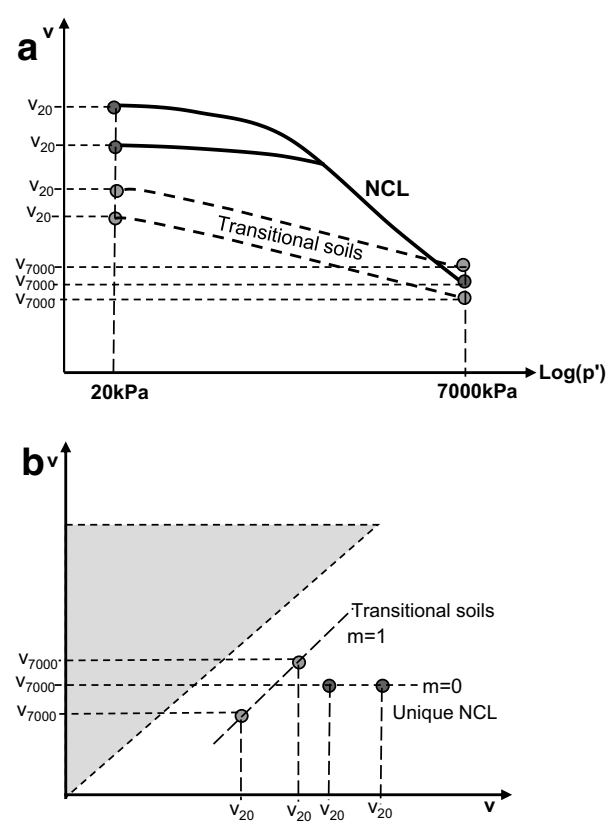

Fig. 6 Quantification of the degree of convergence. a Schematic plot of compression curves and $\mathbf{b}$ calculation of $\mathrm{m}$ (Modified after Ponzoni et al. [19])

mixtures of different soils $[6,12,22]$. However, this behaviour is different from the study of Coop and Atkinson [4] which added relatively close amount of fines (24\%) to sand. The difference can be attributed to the nature of sand which is carbonate (very angular with high initial specific volume) and fines content, which is also non-plastic.

Attempt is made to quantify the degree of convergence of the compression curves using the method proposed by Ponzoni et al. [19] as presented in Fig. 6. The initial specific volume may be plotted against the final specific volume at the highest stress reached in the tests. In Ponzoni et al. [19] study, the initial v was taken at $20 \mathrm{kPa}\left(\mathrm{v}_{20}\right)$ for consistency and the final $\mathrm{v}$ was taken at $6000 \mathrm{kPa}\left(\mathrm{v}_{6000}\right)$, being the highest stress 
reached in the tests. The gradient of the data on the graph denoted as parameter $\mathrm{m}$ is used to describe the degree of convergence. However, the maximum stress reached in this work is approximately $7000 \mathrm{kPa}$, therefore $\mathrm{v}_{7000}$ is used (Fig. 6a). The unshaded area is the permitted space for the data (Fig. 6b). For the soil in which the compression paths are fully convergent, for example uniform sand [5], the $m$ value defined as the gradient on the graph would be zero. Also, $m$ value would be one $(m=1)$ for the soils in which the compression paths are perfectly parallel.

Figure 7 presents the quantification of convergence for different mixtures of the CDV. The degree of convergence $m$ is shown on each plot for different mixtures. The $\mathrm{m}$ value varies for different mixtures and similar behaviour is seen for different mixtures of the HDV which may possibly be the general trend for decomposed volcanic rocks. Different mixtures of the same weathering degree are combined together as presented in Fig. 8. Positive m values ( 0.26 and 0.23 for the CDV and HDV
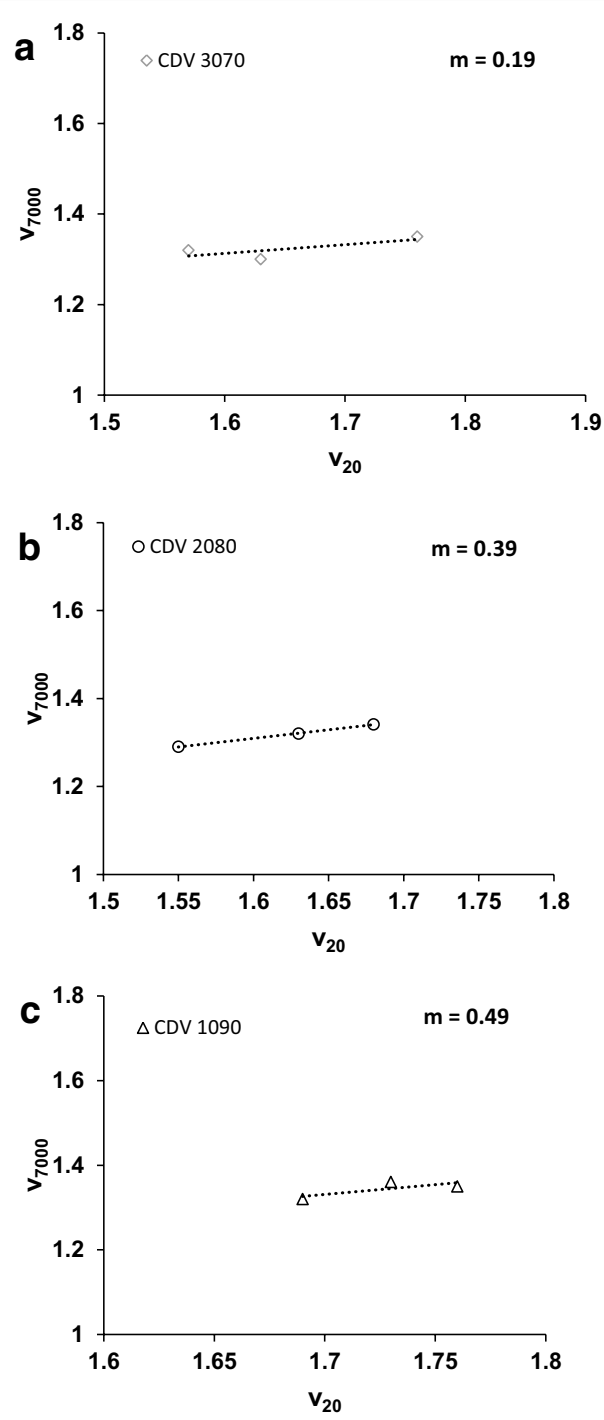

Fig. 7 Typical quantification of convergence for different fines content of CDV. a 3070, b 2080 and c 1090 

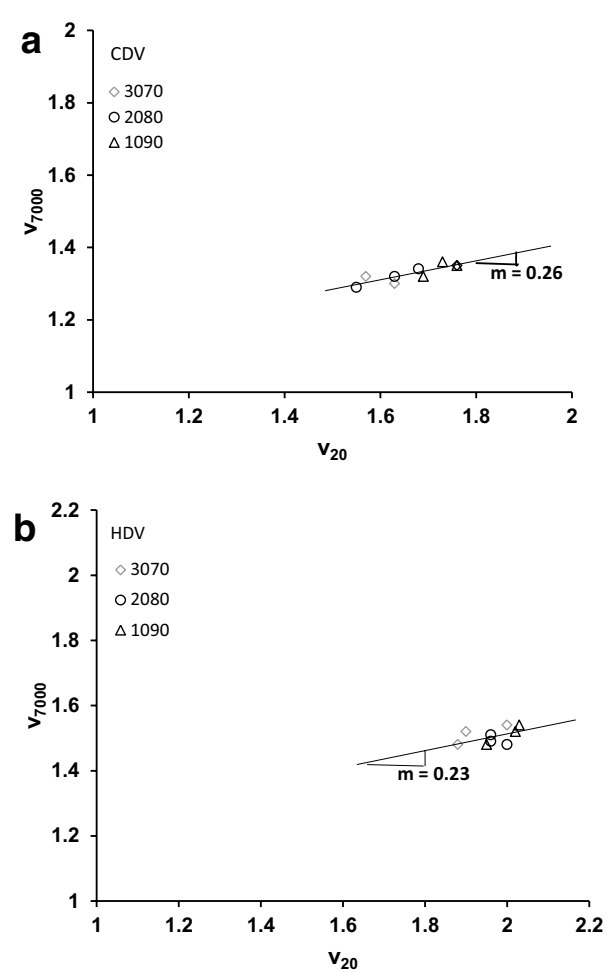

Fig. 8 Combined quantification of convergence: $\mathbf{a}$ CDV mixtures and $\mathbf{b}$ HDV mixtures

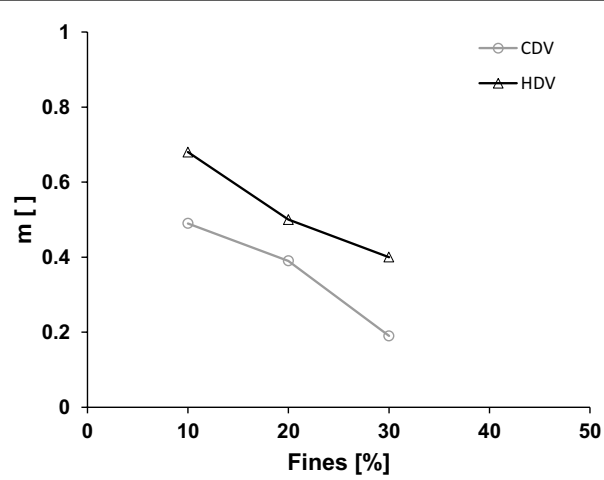

Fig. 9 Variation of degree of convergence with fines content

respectively) are found, which indicates lack of convergence of compression paths to a unique line. The value of $\mathrm{m}$ for the soils from the two weathering degrees is very close. Based on the classification suggested by Ponzoni et al. [19], the samples have a medium degree of convergence similar to what was found for sediments of Venice lagoon.

Figure 9 shows the variation of $m$ values with fines content for the two degrees of weathering. The $\mathrm{m}$ value is reducing with fines, indicating the reduction in the transitional mode of behaviour as the fines content is increasing. Since similar behaviour 
is found for both weathering degree, again this may possibly be the general trend for decomposed volcanic rocks.

In order to shed more light on the mechanisms of compression behaviour of the soils, the particle breakage was quantified using relative breakage $(\mathrm{Br})$ proposed by Hardin [8]. The relative breakage $(\mathrm{Br}=\mathrm{Bt} / \mathrm{Bp})$ is defined as the ratio of the total breakage $(\mathrm{Bt})$, which is the area between the grading curves before and after the test considering only the part above $0.074 \mathrm{~mm}$, to the breakage potential (Bp), which is the area between the initial grading curve and a cut-off at $0.074 \mathrm{~mm}$ (Fig. 10). Figures 11 and 12 present changes in grading curves after compression for samples of the HDV and CDV. This shows that particle breakage occurs during compression. This is in contrast to well graded soils with transitional behaviour studied by Nocilla et al. [13] which had no measurable breakage and also, it is in agreement with soils with non-convergent behaviour studied by Shipton and Coop [22] which had considerable particle breakage. This shows that particle breakage might not be a prerequisite for soils being transitional or not. The offset between initial grading and the grading after the test is reducing with fines, indicating the reduction in particle breakage as the fines increases and sand fraction reduces.

Some studies have found that the particle breakage depends not only on the vertical or mean effective stress reached but also on the initial specific volume (e.g. [5]). Rocchi and Coop [21] found the same trend for the tests at the same maximum stress for their study on well graded saprolitic soils. Also, they stated that the particles in a loose state will have lesser contact points between each other and therefore experience larger stresses. The initial specific volumes in this work ranged between 1.57 and 2.13, and the tests are presented at the highest stress level that can be reached. The trend of particle breakage with average initial specific volume is presented in Fig. 13. The data are scattered because of different initial $\mathrm{v}$ that were achieved for different degrees of weathering, therefore comparison cannot be made for the trend of breakage with initial $\mathrm{v}$ like the other studies. The HDV plots lower than the CDV, therefore indicating that the breakage is less for the less weathered soils. Despite the fact that the particle size distributions are similar for the same soil of different degrees of weathering, the breakage undergone by the soil is different and seems slightly increasing with $\mathrm{v}$ (Fig. 13).

It has been observed that maximum grain size seems to dominate breakage rather than the amount of fines in a mixture [27]. For the granitic saprolites, the particle breakage has been linked to the quantity of large particles and their characteristics [21]. For

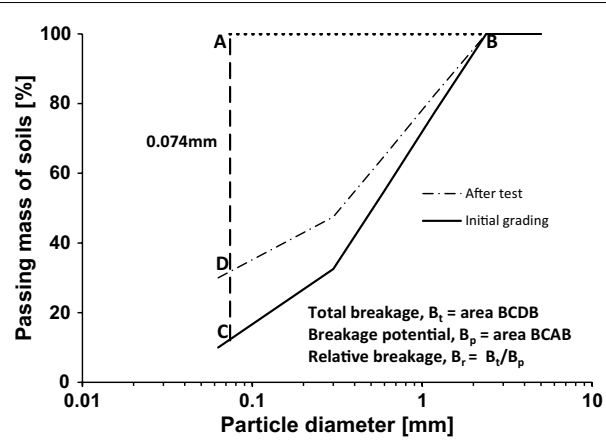

Fig. 10 Definition of relative breakage $B_{r}$ (Modified after [8]) 

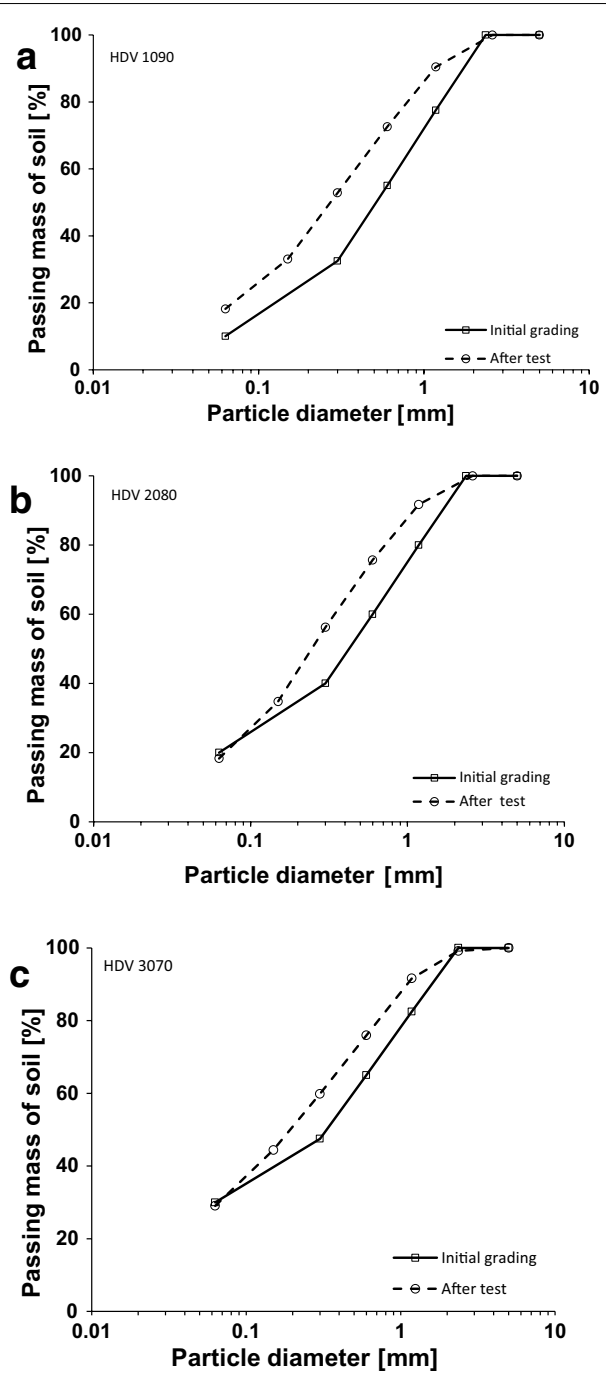

Fig. 11 Change in grading resulting from particle breakage after compression for the HDV. a 1090, b 2080 and $\mathbf{c} 3070$

the samples presented in this paper, the maximum grain size is the same (Fig. 2), therefore particle breakage is studied in relation to fines content and mean particle sizes. The larger value of particle breakage for the CDV (Fig. 14) is as a result of the degree of weathering as well as overall original grading which is coarser for the CDV. Although both CDV and HDV have the same initial artificial gradings but the overall original grading is different as presented in Fig. 2. Figure 14 presents the variation of grading descriptors (fines content and mean sizes $\left(D_{50}\right)$ ) with particle breakage. The effect of fines on the particle breakage is shown in Fig. 14a and the relative breakage reduces with increasing fines for different sample mixtures. Also, the relative breakage increases with the mean particle sizes (Fig. 14b).

In order to show the influence of grain size on the particle breakage for the CDV and HDV, the microstructures of the samples are presented in Fig. 15. The SEM images are of the same field of view and magnification for proper comparison. A closer look at the 

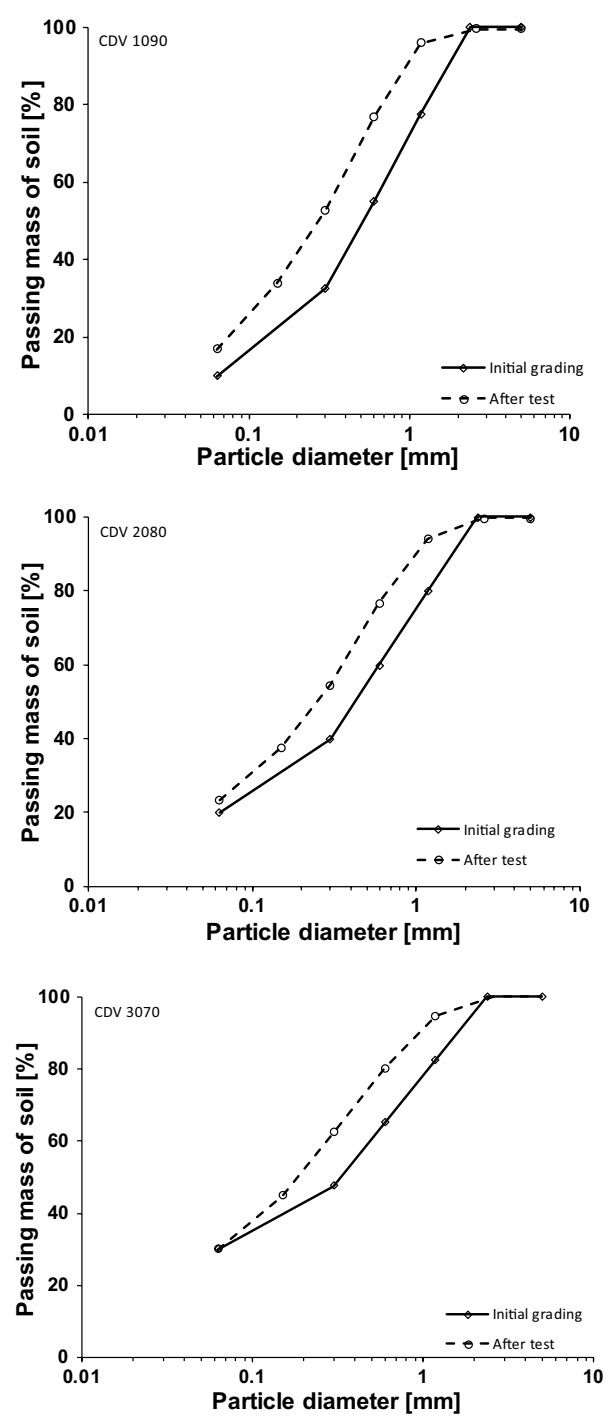

Fig. 12 Change in grading resulting from particle breakage after compression for the CDV

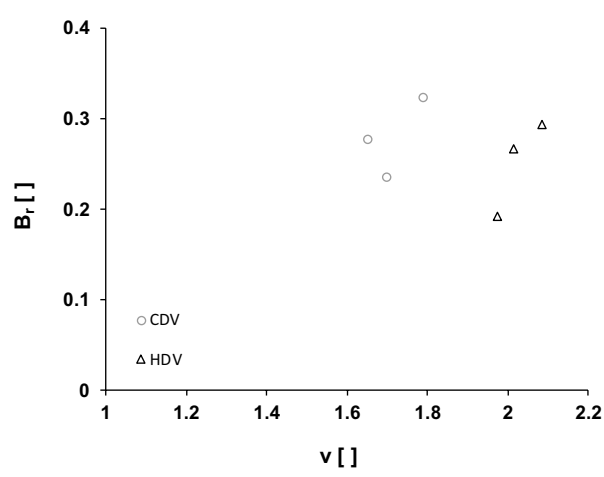

Fig. 13 Variation of particle breakage with specific volume 

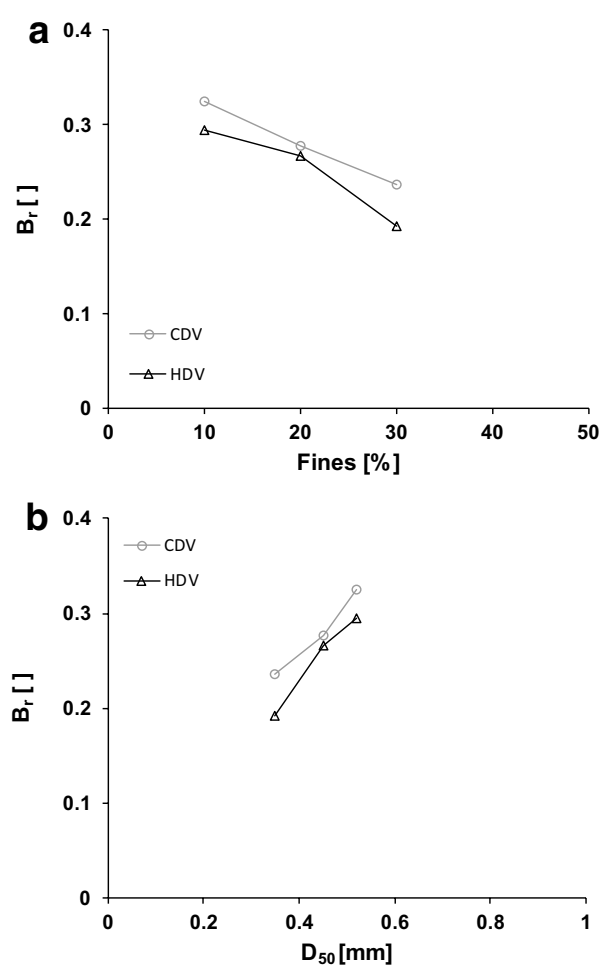

Fig. 14 Variation of particle breakage with grading descriptors. a Fines content and $\mathbf{b}$ mean particle size
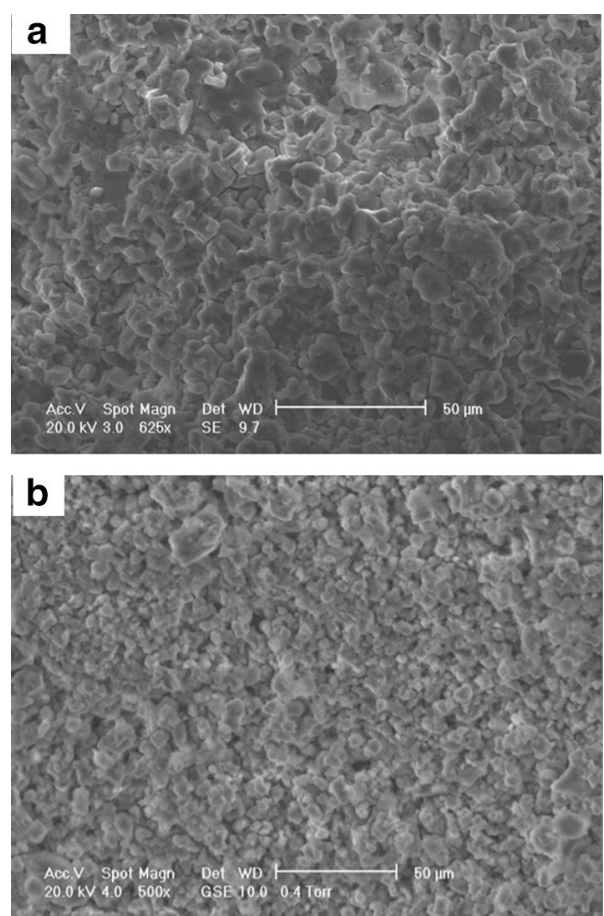

Fig. 15 Microstructure of the samples; a CDV sample and $\mathbf{b}$ HDV sample 


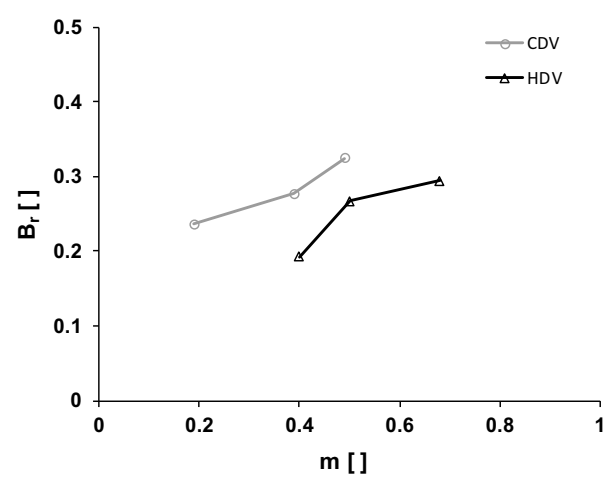

Fig. 16 Influence of particle breakage on the degree of convergence

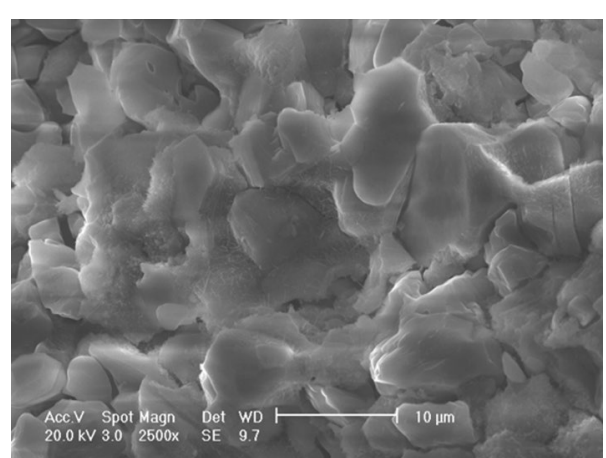

Fig. 17 SEM image showing the particle shape for the CDV

microstructures (Fig. 15) shows that the particles of the CDV are larger than those of the HDV which is in conformity with PSD presented in Fig. 2. Generally, soil with more larger fractions has greater breakage, indicating that the breakage may be linked to the larger particles similar to what was found for granitic saprolites [21]. Comparing the two degrees of weathering, the breakage is higher in the samples of the CDV than those of the HDV. This shows that the more weathered particles may be weaker.

Figure 16 presents the influence of particle breakage on the degree of convergence for the samples. The breakage increases as the degree of convergence $(\mathrm{m})$ increases, that is, the breakage increases with non-convergent behaviour for both the CDV and HDV. This shows that the breakage does not reduce the transitional behaviour in decomposed volcanics, which agrees with what has been found that the breakage of particles does not necessarily means the soils will have a convergent behaviour or not [22].

Particle shape has been found to have great influence on the mechanical behaviour of soils particularly coarse granular soils (e.g., [3, 25]. Okewale [14] investigated the particle shape in relation to elastic modulus at very small strain for decomposed volcanic rocks of the same weathering degree used in this work but the sample was retrieved from different location. The particle shape was determined quantitatively using three descriptors namely, sphericity $(S)$, roundness $(R)$ and regularity $(\rho)$, which 
is the arithmetic mean of $\mathrm{S}$ and $\mathrm{R}$. The particles were found to be angular with almost the same overall regularity for different decomposed volcanic soils.

Figure 17 presents the SEM image of CDV sample used in this study at higher magnification showing the particle shapes. A closer look at the micrograph shows the clusters of particles but the individual particles are angular. However, SEM image of the same magnification for the HDV is not available but from Fig. 3b, it can be seen that HDV particles are also angular, indicating that the particle shapes are similar. In order to confirm the particle shape in a numerical way, the particle shape is determined using regularity by employing the empirical chart proposed by Krumbein and Sloss [10] and modified by Payan et al. [17]. The repeatability of the test was determined by selecting 25 particles in a random manner from the SEM image of each of the CDV and HDV. The mean values of regularity for the CDV and HDV are 0.516 and 0.524 respectively, indicating that the particle shapes are similar. This is also similar to what has been found for similar soils studied by Okewale [14].

\section{Conclusions}

The mechanics of effects of fines on the behaviour of decomposed volcanic rocks have been studied. This was achieved by conducting one-dimensional compression tests for samples of two degrees of weathering. The oedometer tests showed that for different fines and sand mixtures studied, a unique one-dimensional normal compression lines (NCLs) cannot be identified. The degree of transitional behaviour is quantified using the factor and classification proposed by Ponzoni et al. [19]. It was found that there is a medium degree of transitional behaviour for the soils of different weathering degrees and the degree of convergence is reducing with increasing fines content.

The mechanism of compression was further studied by analysing the breakage undergone by the soils. Different particle breakages were found for the soils of similar gradings and this can be attributed to different degrees of weathering. Analysing the breakage further, it was observed that the breakage is more in soil with larger particles (coarser fractions) which constitute the majority of the soil by mass before the test. In addition, it was found that the relative breakage reduces with increasing fines. The breakage is higher in more weathered soil, indicating that the particles of the more weathered volcanic soils may be weaker. The shape of the particles is angular and similar for both decomposed volcanic rocks.

\section{Abbreviations}

Bp: breakage potential; Br: relative breakage; Bt: total breakage; $\mathrm{D}_{50}$ : mean particle size; e: void ratio; LL: liquid limit; $\mathrm{m}$ : parameter for degree of transitional behaviour from oedometer tests data; NCL: normal compression lines; $p^{\prime}$ : mean effective stress; PI: plasticity index; PL: plastic limit; R: roundness; S: sphericity; v: specific volume; $\rho$ : regularity.

Authors' contributions

The author read and approved the final manuscript.

Author details

${ }^{1}$ Federal University of Technology Akure, P.M.B 704, Akure, Nigeria. ${ }^{2}$ Formerly City University of Hong Kong, Tat Chee Avenue, Kowloon, Hong Kong.

\section{Acknowledgements}

The work described in this paper was fully supported by a grant from the Research Grants Council (RGC) of the Hong Kong Special Administrative Region (HKSAR), China (T22-603/15 N). The author also thanks the Civil Engineering \& Development Department (CEDD) of HKSAR for providing the samples used for this research. 


\section{Competing interests}

The authors declare that they have no competing interests.

\section{Publisher's Note}

Springer Nature remains neutral with regard to jurisdictional claims in published maps and institutional affiliations.

Received: 26 September 2018 Accepted: 12 March 2019

Published online: 16 March 2019

\section{References}

1. BS:1377 (1990) Methods of test for soils for civil engineering purposes. British Standard Institution, London

2. Carrera A, Coop MR, Lancellotta R (2011) Influence of grading on the mechanical behaviour of Stava tailings. Geotechnique 61:935-946

3. Cho GC, Dodds J, Santamarina JC (2006) Particle shape effects on packing density, stiffness and strength: natural and crushed sands. J Geotech Geoenviron Eng 132:591-602

4. Coop MR, Atkinson JH (1993) The mechanics of cemented carbonate sands. Geotechnique 43:53-67

5. Coop MR, Lee IK (1993) The behaviour of granular soils at elevated stresses. In: Houlsby GT, Schofield AN (eds) Predictive soil mechanics. Proc. of the C. P. wroth memorial symposium. Thomas Telford, London, pp 186-198

6. Ferreira PMV, Bica AVD (2006) Problems on identification of the effects of structure and critical state in a soil with a transitional behaviour. Geotechnique 56:445-454

7. GEO (Geotechnical Engineering Office) (1988) Guide to rock and soil descriptions. Geoguide 3, Hong Kong Geotechnical Engineering Office

8. Hardin BO (1985) Crushing of soil particles. J Geotech Geoenviron Eng 111:1177-1192

9. ISRM (2007) The complete ISRM suggested methods for rock characterisation, testing and monitoring [1974-2006]. In: Ulusuay R, Hudson J (eds) International society of rock mechanics

10. Krumbein WC, Sloss LL (1963) Stratigraphy and sedimentation, 2nd edn. Freeman and Company, San Francisco

11. Lade JA, Yamamuro PV (1997) Static liquefaction of very loose sands. Can Geotech J 34:905-917

12. Martins F, Bressani LA, Coop MR, Bica VD (2001) Some aspects of the compressibility behaviour of a clayey sand. Can Geotech J 38:1177-1186

13. Nocilla A, Coop MR, Colleselli F (2007) The mechanics of Italian Silt; an example of transitional behaviour. Geotechnique 56(4):261-271

14. Okewale IA (2017) Geotechnical and geological characterisation of decomposed volcanic rocks from Hong Kong. City University of Hong Kong, Ph.D thesis

15. Okewale IA, Coop MR (2017) A study of the effects of weathering on soils derived from decomposed volcanic rocks. Eng Geol 222:53-71

16. Okewale IA, Coop MR (2018) Suitability of different approaches for analysing and predicting the behaviour of decomposed volcanic rocks. J Geotech Geoenviron Eng 144(01048064s):1-14

17. Payan M, Khoshghalb A, Senetakis K, Nasser K (2016) Effect of particle shape and validity of $G_{\max }$ models for sand: a critical review and a new expression. Comput Geotech 72:28-41

18. Ponzoni E, Nocilla A, Coop MR (2017) The behaviour of a gap graded sand with mixed mineralogy. Soils Found 57:1030-1044

19. Ponzoni E, Nocilla A, Coop MR, Colleselli F (2014) Identification and quantification of transitional modes of behaviour in sediments of Venice lagoon. Geotechnique 64:694-708

20. Rocchi I, Coop MR (2014) Experimental accuracy of the initial specific volume. Geotech Test J 37:168-175

21. Rocchi I, Coop MR (2016) Mechanisms of compression in well-graded saprolitic soils. Bull Eng Geol Environ. https:// doi.org/10.1007/s10064-015-0841-7

22. Shipton B, Coop MR (2012) On the compression behaviour of reconstituted soils. Soils Found 52:668-681

23. Ventouras K, Coop MR (2009) On the behaviour of Thanet sand: an example of an uncemented natural sand. Geotechnique 59:727-738

24. Xiao Y, Coop MR, Liu H, Liu H, Jiang JS (2016) Transitional behaviours in well-graded coarse granular soils. J Geotech Geoenviron Eng 142:06016018

25. Xiao Y, Long L, Evans TM, Zhou H, Liu H, Stuedlein AW (2019) Effect of particle shape on stress-dilatancy responses of medium-dense sands. J Geotech Geoenviron Eng 145:04018105

26. Yin JH (1999) Properties and behaviour of Hong Kong marine deposits with different clay contents. Can Geotech J 36:1085-1095

27. Zhang X, Baudet BA (2013) Particle breakage in gap-graded soils. Geotech Lett 3:72-77

28. Zuo L, Baudet BA (2015) Determination of the transitional fines content of sand-non plastic fines mixtures. Soils Found 55:213-219 